\title{
Human phase I metabolism of the novel synthetic cannabinoid 5F-CUMYL-PEGACLONE
}

\author{
Lukas Mogler $^{1,2,3} \cdot$ Sebastian Halter ${ }^{1,2,3} \cdot$ Maurice Wilde $^{1,2,3} \cdot$ Florian Franz $^{1,2,3} \cdot$ Volker Auwärter $^{1,3}$
}

Received: 23 August 2018 / Accepted: 24 September 2018 / Published online: 5 October 2018

(c) The Author(s) 2018

\begin{abstract}
Purpose 5F-CUMYL-PEGACLONE is a recently emerged $\gamma$-carbolinone derived synthetic cannabinoid. The present study aimed to identify phase I metabolites to reliably prove consumption of the substance by urine analysis and to differentiate from the uptake of the non-fluorinated analog CUMYL-PEGACLONE.

Methods For metabolite characterization, phase I metabolites were analyzed by liquid chromatography-high resolution mass spectrometry after incubation with pooled human liver microsomes. Reliability of the biomarkers was evaluated by analysis of human urine samples $(n=20)$ by liquid chromatography-triple quadrupole tandem mass spectrometry. Sample preparation included $\beta$-glucuronidase treatment followed by liquid-liquid extraction.

Results In total, 15 metabolites were detected in vivo and characterized. Metabolic reactions were primarily observed at the $\gamma$-carbolinone core and the 5-fluoropentyl chain, and included $N$-dealkylation, hydroxylation, hydrolytic defluorination, formation of a dihydrodiol, oxidation to the pentanoic acid metabolite and formation of the propionic acid metabolite. Six of these metabolites were identical with phase I metabolites of CUMYL-PEGACLONE, which must be considered for interpretation of analytical findings in urine samples.

Conclusions 5F-CUMYL-PEGACLONE was subject to extensive metabolism in humans. The propionic acid metabolite was the most abundant metabolite in all urine samples and should be targeted when maximum sensitivity is needed (e.g., drug abstinence control). However, this metabolite also occurs in the biotransformation of the non-fluorinated analog and is, therefore, not a compound-specific marker. For differentiation, a metabolite hydroxylated at the $\gamma$-carbolinone core showed to be the most reliable marker and should be used as an additional target analyte.
\end{abstract}

Keywords 5F-CUMYL-PEGACLONE $\cdot$ Metabolism in vivo and in vitro $\cdot \gamma$-Carbolinone $\cdot$ Human liver microsomes · Urine analysis $\cdot$ 5F-SGT-151

\section{Introduction}

Electronic supplementary material The online version of this article (https://doi.org/10.1007/s11419-018-0447-4) contains supplementary material, which is available to authorized users.

Volker Auwärter

volker.auwaerter@uniklinik-freiburg.de

1 Institute of Forensic Medicine, Forensic Toxicology, Medical Center-University of Freiburg, Albertstraße 9, 79104 Freiburg, Germany

2 Hermann Staudinger Graduate School, University of Freiburg, Hebelstraße 27, 79104 Freiburg, Germany

3 Faculty of Medicine, University of Freiburg, Breisacherstrasse 153, 79110 Freiburg, Germany
Synthetic cannabinoids (SCs) are a class of designer drugs recreationally used to mimic the effects of cannabis. Among the new psychoactive substances (NPS), SCs are still among the largest subgroups monitored by the European Monitoring Centre for Drugs and Drug Addiction (EMCDDA). According to recent reports from July 2018, there were 179 different SCs on the European drug market [1]. SCs were detected in "legal high" products in 2008 for the first time [2, 3]. Since then, clandestine laboratories synthesized compounds often based on pharmaceutical research papers and patent applications $[4,5]$. For instance, several highly potent SCs that emerged on the drug market were first described in a patent application of Bowden and Williamson ("SGT-compounds") [6]. These drugs are characterized by a cumyl substituent, 


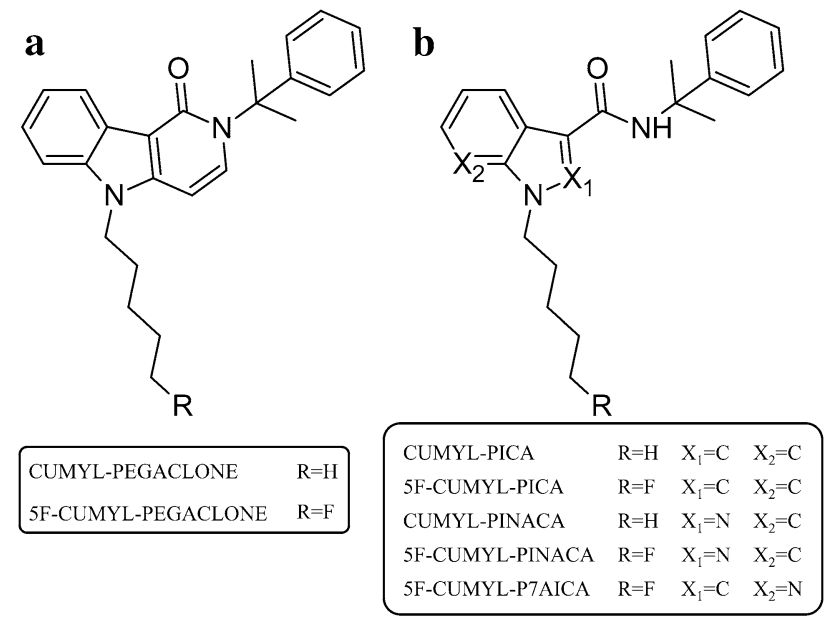

Fig. 1 Structures of a $\gamma$-carbolinone derived cumyl synthetic cannabinoids (SCs), and $\mathbf{b}$ indole, indazole or azaindole derived cumyl carboxamide type SCs

which is attached to indole, indazole or azaindole core structures by a linker moiety (Fig. 1). Pharmacological evaluation of some of these substances showed that cumyl derived SCs are potent agonists at the cannabinoid receptors $\mathrm{CB}_{1}$ and $\mathrm{CB}_{2}[7,8]$. The first cumyl-carrying SC comprising a $y$-carbolinone core structure occurred on the German drug market in December 2016. CUMYL PEGACLONE (5-pentyl-2-(2phenylpropan-2-yl)-2,5-dihydro-1 $H$-pyrido[4,3- $b$ ]indol-1one) (Fig. 1) was identified in an herbal mixture and was also sold under the street name SGT-151. The compound showed full agonistic properties with binding affinities in the low nanomolar range at $\mathrm{CB}_{1}$ and $\mathrm{CB}_{2}[9,10]$. The human phase I metabolism of CUMYL-PEGACLONE was recently described [10,11]. In 2018, a substance purchased as a "research chemical" online was identified as 5F-CUMYLPEGACLONE (5-(5-fluoropentyl)-2-(1-methyl-1phenylethyl)-1H-pyrido[4,3-b]indol-1-one) (Fig. 1) in our laboratory. Exchange of a hydrogen atom of the $N$-pentyl side chain by a fluorine atom at $\mathrm{C}_{5}$ has been a common modification also applied to other core structures by clandestine chemists $[12,13]$.

To prove the uptake of SCs, urine is often the preferred biological matrix for forensic and clinical toxicology, particularly in drug abstinence testing when longer detection windows are needed. In urine, metabolites of SCs are the target compounds as parent SCs are rarely detectable due to extensive metabolism. To enhance sensitivity, cleavage of phase II conjugates is a common step prior to liquid chromatography-mass spectrometry (LC-MS) analysis $[14,15]$. The major problem in urine analysis of SC metabolites is the lack of commercially available reference standards. To circumvent this problem, in vitro generation of phase I metabolites, e.g., by human liver microsomes (HLMs), human hepatocytes, or even fungi was established [16-18]. Tentative identification and characterization of the metabolites are commonly carried out by high resolution mass spectrometry (HRMS) techniques that allow identification based on accurate mass and isotopic patterns. For metabolite screening in urine, liquid chromatography-tandem mass spectrometry (LC-MS/MS) methods are still a frequently used alternative to HRMS in forensic and clinical toxicology when maximum sensitivity for routine purposes is required [19].

The aim of the present study was to tentatively identify and characterize human phase I metabolites produced in an HLM assay by liquid chromatography-quadrupole time-offlight mass spectrometry (LC-QToF-MS). These data were compared with the phase I metabolite profile detected in urine samples from drug users by an LC-MS/MS screening method where at least two metabolites were detected. Based on these results, phase I metabolites were evaluated as targets for urine screening to reliably prove 5F-CUMYL-PEGACLONE consumption and to differentiate from the uptake of the non-fluorinated analog CUMYL-PEGACLONE.

\section{Materials and methods}

\section{Chemicals and reagents}

Formic acid (Rotipuran ${ }^{\circledR} \geq 98 \%$, p.a.) and potassium hydrogen phosphate $(\geq 99 \%$, p.a.) were obtained from Carl Roth (Karlsruhe, Germany); acetonitrile (ACN) (LC-MS grade), ammonium formate $10 \mathrm{M}(99.995 \%)$, potassium hydroxide [puriss. p.a. $\geq 86 \%$ (T) pellets] and superoxide dismutase (SOD) ( $\geq 3000$ units/mg protein from bovine erythrocytes) from Sigma-Aldrich (Steinheim, Germany); pooled HLMs (50 donors, $20 \mathrm{mg} / \mathrm{mL}$ protein in $250 \mathrm{mM}$ sucrose), NADPH regenerating solutions A and B (reductase activity $0.43 \mu \mathrm{mol} / \mathrm{min} / \mathrm{mL}$ ), and potassium phosphate buffer $0.5 \mathrm{M}$ (pH 7.5) from Corning (Corning, NY, USA); $\beta$-glucuronidase (E. coli K 12) from Roche Diagnostics (Mannheim, Germany). Mobile phase A (1\% v/v ACN, $0.1 \% \mathrm{v} / \mathrm{v} \mathrm{HCOOH}, 2 \mathrm{mM}$ ammonium formate in water) and mobile phase B $(0.1 \%$ v/v HCOOH, $2 \mathrm{mM}$ ammonium formate in $\mathrm{ACN}$ ) were freshly prepared prior to analysis. 5F-CUMYL-PEGACLONE and CUMYL-PEGACLONE reference standards were obtained from Chiron AS (Trondheim, Norway). Stock solutions $(1 \mathrm{mg} / \mathrm{mL})$ were prepared in $\mathrm{ACN}$ and stored at $-20{ }^{\circ} \mathrm{C}$ until analysis.

\section{Pooled human liver microsome assay}

Total assay volume of $100 \mu \mathrm{L}$ consisted of $5 \mu \mathrm{L}$ pooled HLM solution, $1 \mu \mathrm{L}$ parent compound stock solution $(1 \mathrm{mg} / \mathrm{mL}$ in $\mathrm{ACN}), 5 \mu \mathrm{L}$ NADPH regenerating solution A, $1 \mu \mathrm{L}$ NADPH regenerating solution $\mathrm{B}, 10 \mu \mathrm{L}$ SOD, $20 \mu \mathrm{L}$ phosphate buffer, 
and $58 \mu \mathrm{L}$ deionized water. Incubation was conducted for $30 \mathrm{~min}$ at $37^{\circ} \mathrm{C}$. The incubation was quenched by adding $100 \mu \mathrm{L}$ of ice-cold ACN. After centrifugation, the supernatant was transferred into a separate vial and stored at $-20{ }^{\circ} \mathrm{C}$. Two negative control samples were processed in the same way. One was performed with $5 \mu \mathrm{L}$ phosphate buffer instead of pooled HLMs and a second with $1 \mu \mathrm{L}$ ACN instead of the substrate. Prior to LC-MS/MS analysis the supernatant was diluted 1:10 in mobile phase A/B (70:30, v/v). For LC-QToF-MS analysis in the electrospray ionization (ESI) mode, $100 \mu \mathrm{L}$ supernatant was evaporated until dryness and reconstituted in $25 \mu \mathrm{L}$ mobile phase $\mathrm{A} / \mathrm{B}(70: 30, \mathrm{v} / \mathrm{v})$.

\section{Human urine samples}

The urine samples $(n=20)$ were collected during LC-MS/ MS routine screening for metabolites of synthetic cannabinoids between April 2018 and July 2018. Samples were rated positive for the consumption of an $\mathrm{SC}$ when at least two metabolites were detected, which met the identification criteria for LC-MS/MS analyses as defined by the German Society of Toxicological and Forensic Chemistry (GTFCh) [19]. Incubations of pooled HLMs and one urine sample (with a corresponding blood sample proving the consumption of 5F-CUMYL-PEGACLONE) served as a positive control. Urine samples positive for CUMYL-PEGACLONE metabolites $(n=6)$ were also used in the previous metabolism study [11]. All urine samples were sent to the laboratory of forensic toxicology in Freiburg (Germany) for drug abstinence control testing and all analyses were conducted in accordance with the inquiry of the respective client.

\section{Urine sample preparation}

An aliquot of $0.5 \mathrm{~mL}$ of urine was treated with $0.5 \mathrm{~mL}$ phosphate buffer ( $\mathrm{pH} \mathrm{6)}$ and $30 \mu \mathrm{L} \beta$-glucuronidase for conjugate cleavage at $45^{\circ} \mathrm{C}$ for $60 \mathrm{~min}$. Liquid-liquid extraction was performed by adding $1.5 \mathrm{~mL} \mathrm{ACN}$ and $0.5 \mathrm{~mL}$ of a $10 \mathrm{M}$ ammonium formate solution. After shaking and centrifugation the organic layer was transferred into a separate vial and evaporated to dryness under a nitrogen stream at $40{ }^{\circ} \mathrm{C}$. Reconstitution was done in $200 \mu \mathrm{L}$ mobile phase A/B (70:30, v/v) prior to LC-MS/MS analysis or in $25 \mu \mathrm{L}$ mobile phase A/B (70:30, v/v) prior to LC-QToF-MS analysis.

\section{LC-ESI-QToF-MS experiments}

LC-ESI-QToF-MS analysis was performed on an impact ${ }{ }^{\mathrm{TM}}$ QToF instrument coupled with an Elute HPLC system (both from Bruker Daltonik, Bremen, Germany). Chromatographic separation was achieved on a Kinetex ${ }^{\circledR} \mathrm{C} 18$ column (2.6 $\mu \mathrm{m}, 100 \AA, 100 \times 2.1 \mathrm{~mm}$; Phenomenex, Aschaffenburg, Germany), protected by an equivalent Security Guard ${ }^{\mathrm{TM}}$
ULTRA catridge precolumn (Phenomenex), applying gradient elution as follows: total LC run time was 15 min with a mobile phase B starting concentration of $30 \%$, linearly increased to $45 \%$ in $9.0 \mathrm{~min}$, further increased to $70 \%$ in $1.0 \mathrm{~min}$, further increased to $95 \%$ in $1.0 \mathrm{~min}$, held for $2.0 \mathrm{~min}$, decreased to starting conditions of $30 \%$ in $0.1 \mathrm{~min}$ and held for $1.9 \mathrm{~min}$ for re-equilibration. The flow rate was set to $0.4 \mathrm{~mL} / \mathrm{min}$. The autosampler was cooled down to $10{ }^{\circ} \mathrm{C}$. Column oven temperature was $40{ }^{\circ} \mathrm{C}$. The injection volume was $10 \mu \mathrm{L}$. HyStar ${ }^{\mathrm{TM}}$ version 3.2 and DataAnalysis version 4.2 (both from Bruker Daltonik) were used for data acquisition and processing, respectively. The QToF-MS was operated in positive ionization mode acquiring spectra in the range of $m / z 30-600$ in full scan (acquisition rate of $4.0 \mathrm{~Hz}$ ), and broadband collision induced dissociation (bbCID) data were acquired in one run. The collision energy applied for bbCID was $30 \pm 6 \mathrm{eV}$. Instrument parameters were set as described previously [13]. CID fragmentation experiments for 5F-CUMYL-PEGACLONE were performed with the reference standard solution at $1 \mu \mathrm{g} / \mathrm{mL}$ with LC-HRMS parameters as stated earlier.

\section{LC-ESI-MS/MS experiments}

LC-ESI-QTRAP-MS analysis was performed with a Nexera X2 UHPLC (Shimadzu, Duisburg, Germany) coupled to a QTRAP $^{\circledR} 5500$ triple quadrupole linear ion trap instrument (SCIEX, Darmstadt, Germany). Chromatographic parameters, injection volume, autosampler and column oven temperature were as described earlier.

The QTRAP-MS was operated with positive ionization in multiple reaction monitoring (MRM) mode and enhanced product ion (EPI) scan mode. The respective potentials (declustering potential (DP), entrance potential (EP), collision energies (CE), and collision cell exit potential (CXP) of the multiple reaction monitoring (MRM) ion transitions of the parent compound were optimized under direct infusion $(10 \mathrm{ng} / \mathrm{mL})$ (see Supplementary Material Table S1).

\section{In vitro metabolite identification and characterization}

Metabolites generated in the pooled HLM assay were tentatively identified and characterized by LC-QToF-MS in manual data processing with following criteria: MS peak area $>1 \times 10^{5} \mathrm{cps}$, mass error of the precursor ion $<5 \mathrm{ppm}$, signal-to-noise ratio $>3: 1$, and mass tolerance for fragment ions $\pm 10 \mathrm{ppm}$. First of all, a list of hypothetical metabolites was generated on the basis of previous metabolism studies of structurally related SCs [11, 20, 21]. To avoid missing the main metabolites, precursor ions were searched via typical fragment ions in the bbCID data. Based on the LC-HRMS data, an LC-MS/MS MRM-method was developed, 
comprising the two most abundant ion transitions of each metabolite applying the optimized MS parameters of the parent compound (see Supplementary Material Table S2). The most abundant fragment ions were identified by recording EPI spectra of the metabolites. For EPI scans, EP was set to $10 \mathrm{~V}$ and a CE of $35 \mathrm{~V}$ with a spread of $\pm 15 \mathrm{~V}$ was applied. The two most abundant in vitro metabolites (M10 and M12) were integrated into an existing LC-MS/MS routine screening method to screen for positive urine samples.

\section{Identification of metabolites in urine samples and biomarker evaluation}

A metabolite was regarded as "identified" in LC-MS/MS analysis of urine samples when the following criteria were met: signal-to-noise ratio $>3: 1$; peak area $>1 \times 10^{4} \mathrm{cps}$; retention time (RT) $( \pm 0.1 \mathrm{~min})$; matching EPI spectra (when in vitro reference spectra where available); metabolites, not detectable in the pooled HLM assay, but additionally confirmed by accurate mass (LC-QToF-MS analysis). In order to compare the relative abundances of the metabolites as a rough estimate of their concentrations, peak area ratios were calculated by dividing the peak area of a detected metabolite in a sample by the peak area of the most abundant metabolite (M06) in the same sample. In this way, the metabolites were ranked by their mean peak area ratios over all analyzed urine samples. The relative standard deviations (RSDs) of the mean peak area ratios provide an estimation of the variability of the metabolites' rank positions within the investigated sample collective.

\section{Results and discussion}

\section{LC-ESI-QToF-MS characterization of 5F-CUMYL-PEGACLONE}

To investigate the ESI-MS fragmentation behavior of 5F-CUMYL-PEGACLONE $\left(\mathrm{C}_{25} \mathrm{H}_{28} \mathrm{FN}_{2} \mathrm{O}^{+} ; \mathrm{m} / z\right.$ 391.2180), a solution of $1 \mu \mathrm{g} / \mathrm{mL}$ was analyzed by LC-ESI-QToF-MS in full scan and bbCID mode. The proposed fragmentation pathways of 5F-CUMYL-PEGACLONE are shown in Fig. 2. CID fragmentation led to the main fragment ion a $\left(\mathrm{C}_{16} \mathrm{H}_{18} \mathrm{FN}_{2} \mathrm{O}^{+} ; m / z 273.1398\right)$, most probably due to $\alpha$-cleavage between the lactam nitrogen of the core system and the benzyl carbon of the cumyl moiety (Fig. 2a).

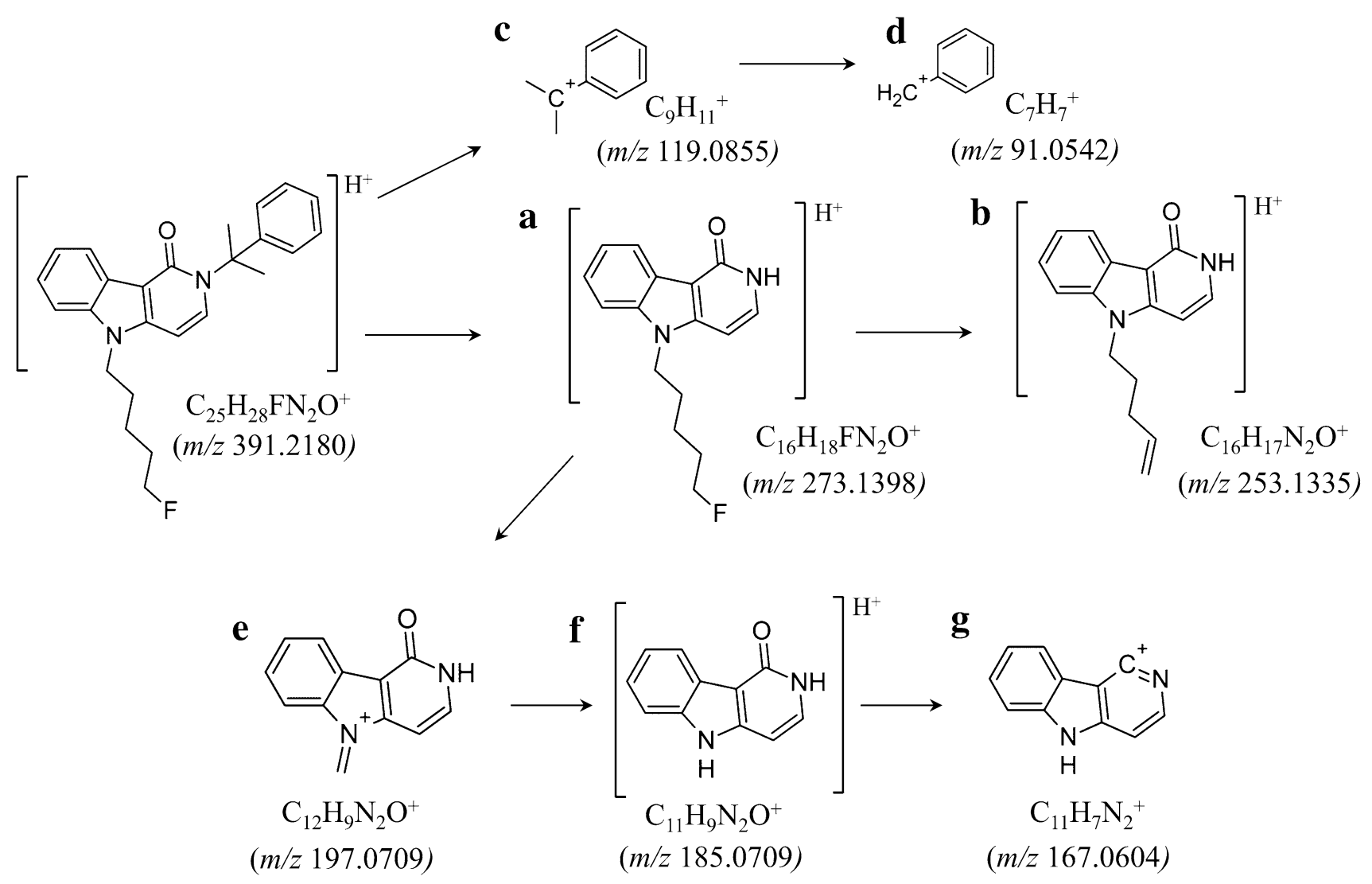

Fig. 2 Proposed pathways for the formation of the main characteristic fragment ions in the liquid chmromatography-electrospray ionizationcollision induced dissociation spectra of 5F-CUMYL-PEGACLONE 
Fragment $\mathbf{b}\left(\mathrm{C}_{16} \mathrm{H}_{17} \mathrm{~N}_{2} \mathrm{O}^{+} ; m / z 253.1335\right)$ is the product of HF elimination of fragment a (Fig. 2b). This pathway has previously been described for SCs with an indazole core ring [22]. Fragmentation of the cumyl moiety leads to the dimethylbenzyl ion $\mathrm{c}\left(\mathrm{C}_{9} \mathrm{H}_{11}{ }^{+} ; \mathrm{m} / z\right.$ 119.0855) (Fig. 2c), which is further degraded by twofold loss of $\mathrm{CH}_{2}$ to the tropylium ion $\mathrm{d}\left(\mathrm{C}_{7} \mathrm{H}_{7}+; m / z\right.$ 91.0542). A characteristic fragmentation pathway for the $\gamma$-carbolinone core is formation of the three fragment ions $\mathrm{e}, \mathrm{f}$ and $\mathrm{g}$ by further fragmentation of fragment ion a (Fig. 2e, f, g). Fragment ion $\mathrm{f}\left(\mathrm{C}_{11} \mathrm{H}_{9} \mathrm{~N}_{2} \mathrm{O}^{+} ; m / z\right.$ 185.0709) is the most prominent fragment of those and represents the $\gamma$-carbolinone ion. Fragment $\mathrm{g}\left(\mathrm{C}_{11} \mathrm{H}_{7} \mathrm{~N}_{2}{ }^{+} ; \mathrm{m} / z\right.$ 167.0604) is most probably formed by the loss of $\mathrm{H}_{2} \mathrm{O}$ from fragment ion f. Comparable degradations of SCs based on tricyclic core systems have been described for carbazole derived SCs [13]. Fragment ion e $\left(\mathrm{C}_{12} \mathrm{H}_{9} \mathrm{~N}_{2} \mathrm{O}^{+} ; \mathrm{m} / z\right.$ 197.0709) is less produced by CID and could be referred to a dealkylation of fragment ion a in allylic position leading to a $\gamma$-carbolinone- $N$-methyl ion. This assumption is supported by the fact that fragment ion e was not detectable in CID spectra of $\mathrm{N}$-dealkylated metabolites. The corresponding spectra can be found in Supplementary Material Fig. S1.

\section{Microsomal phase I metabolism of 5F-CUMYL-PEGACLONE}

In the pooled HLM assay, 30 phase I metabolites were generated and characterized. None of the metabolites were detected in the control samples. The observed metabolic reactions in vitro were hydroxylation, hydrolytic defluorination, $\mathrm{N}$-dealkylation, aldehyde/ketone formation, formation of a dihydrodiol, oxidation to the carboxylic acid and combinations thereof. A detailed list of the detected in vitro metabolites can be found in the Supplementary Material (Table S3). To update an existing LC-MS/MS screening method, the ion transitions of the most abundant in vitro metabolites (in vivo confirmed as M10 and M12) were integrated. Subsequently, 20 urine samples were found positive for these metabolites, which met the set identification criteria [19].

\section{Human in vivo phase I metabolites of 5F-CUMYL-PEGACLONE}

In total, 15 different phase I metabolites were detected in the investigated set of urine samples. The metabolic reactions in vivo included hydroxylation, formation of a dihydrodiol, hydrolytic defluorination, $\mathrm{N}$-dealkylation, oxidation to the pentanoic acid metabolite, side chain degradation to the propionic acid metabolite and combinations thereof. The metabolites were named serially numbered in the order of their retention times (RTs). The parent compound was not detected in any of the urine samples. Since the identification of the particular position of functional groups introduced by metabolic reactions would require isolation of metabolites for structure elucidation, e.g., by nuclear magnetic resonance (NMR) spectroscopy or synthesis of reference material, the exact chemical structures of some metabolites remain unclear. Table 1 shows the detected in vivo phase I metabolites with the proposed biotransformation step, tentative localizations of the metabolic modifications, detected $[\mathrm{M}+\mathrm{H}]^{+}$precursor masses including their mass errors, elemental compositions, characteristic fragment ions including their mass errors and the overall rank position. The product ion mass spectra for localization of metabolic modification are shown in Supplementary Material Table S4. Accurate masses were obtained from signals of the in vitro assay.

The metabolites M11-M15 with $[\mathrm{M}+\mathrm{H}]^{+}$at $m / z 407.2129$ are formed by hydroxylation within human phase I metabolism. M11 and M12 are monohydroxylated at the 5-fluoropentyl chain with the characteristic product ions at $\mathrm{m} / \mathrm{z}$ 289.1347, 197.0709 and unaltered cumyl moiety fragments $c$ and d. The hydroxy group of M13 and M15 could be located at the $\gamma$-carbolinone core, indicated by the fragment ion at $\mathrm{m} / \mathrm{z} 289.1347$ in combination with the diagnostic core fragments at $\mathrm{m} / z 201.0659$ and 183.0553. M14 showed the fragment ions at $m / z 273.1398135 .11686$, suggesting a hydroxylated cumyl moiety.

Further hydroxylation led to the dihydroxylated metabolites M03 and M05 with $[\mathrm{M}+\mathrm{H}]^{+}$at $\mathrm{m} / z$ 423.2078. Both metabolites are hydroxylated at the core system and the 5 -fluoropentyl chain yielding in diagnostic product ions at $\mathrm{m} / \mathrm{z} 305.1296,213.0659$, and unaltered cumyl moiety fragment $\mathrm{c}$.

Monohydroxylation in combination with $N$-dealkylation of the 5-fluoropentyl chain led to the metabolites M01 and M02 ([M+H $]^{+}$at $\left.m / z 319.1441\right)$. The hydroxylation occurs at the $\gamma$-carbolinone core with fragment ions at $m / z 201.0659$ and 183.0553 .

M10 is formed by hydrolytic defluorination with $[\mathrm{M}+\mathrm{H}]^{+}$ at $m / z 389.2224$ and diagnostic product ions at 271.1441, with unaltered fragment ions $\mathrm{c}$ and $\mathrm{d}$ for the cumyl moiety as well as unaltered fragment ions e and $\mathrm{f}$ for the $\gamma$-carbolinone core. Oxidation of M10 most likely led to the pentanoic acid metabolite M08 with $[\mathrm{M}+\mathrm{H}]^{+}$at $\mathrm{m} / \mathrm{z}, 403.2016$. The diagnostic product ion at $\mathrm{m} / z 285.1234$ followed by elimination of $\mathrm{CO}_{2}$ (fragment ion at $\mathrm{m} / z$ 239.1179) exclude that this metabolite is formed by ketone formation in combination with hydroxylation, which was described as a major metabolite for CUMYL-PEGACLONE [11].

Oxidative degradation of the 5-fluoropentyl chain led to the propionic acid metabolite of M08 with $[\mathrm{M}+\mathrm{H}]^{+}$at $\mathrm{m} / \mathrm{z} 375.1703$ (M06). The characteristic fragment ion at $\mathrm{m} / \mathrm{z}$ 257.0921 and the unaltered fragment ions $\mathrm{c}$ and $\mathrm{d}$ for the cumyl moiety as well as unaltered fragment ion e for the $\gamma$-carbolinone core were detected. M06 was not detected in 


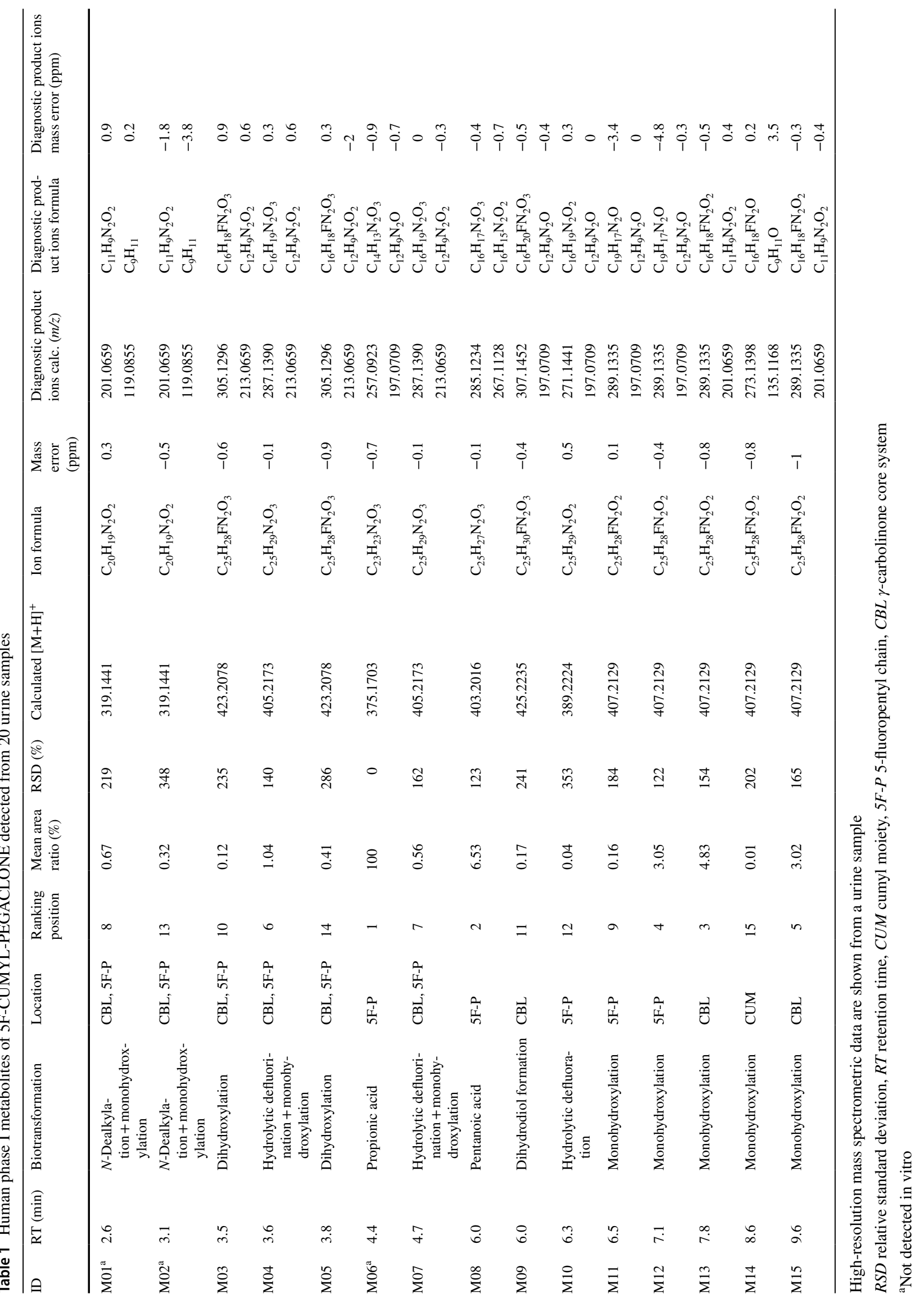


the pooled HLM assay, but turned out to be the most abundant metabolite of 5F-CUMYL-PEGACLONE in human phase I metabolism under the chosen analytical conditions. A similar biotransformation producing a highly abundant propionic acid metabolite has already been described for the SC 5F-MDMB-PICA [15].

Monohydroxylation in combination with hydrolytic defluorination generated $\mathrm{M} 04$ and $\mathrm{M} 07$ with $[\mathrm{M}+\mathrm{H}]^{+}$at $\mathrm{m} / \mathrm{z}$ 405.2173 and a characteristic product ion at $\mathrm{m} / \mathrm{z} 287.1390$. The hydroxylation site was the $\gamma$-carbolinone core with fragment ions at $\mathrm{m} / z 201.0659$ and 183.0553 .

The metabolite M09 with $[\mathrm{M}+\mathrm{H}]^{+}$at $\mathrm{m} / z 425.2235$ could be referred to the formation of a dihydrodiol functionality at the $\gamma$-carbolinone core with diagnostic product ions at $m / z$ 307.1452 and the unaltered cumyl fragment $\mathrm{c}$ most probably formed by hydrolysis after epoxidation.

The postulated phase I metabolic pathways of 5F-CUMYL-PEGACLONE in humans are shown in Fig. 3.

\section{Comparison of in vivo and in vitro results}

Twelve of the detected in vivo metabolites could be confirmed by corresponding signals in pooled HLM samples. The in vivo metabolites M01 and M02, both $N$-dealkylated and monohydroxylated at the core system, could not be detected in the HLM assay. This might be explained by a relatively weak tendency of HLMs to perform multiple biotransformations under the chosen conditions. The most abundant in vivo metabolite was the propionic acid metabolite. This metabolite is most probably formed by $\beta$-oxidation of the pentanoic acid metabolite. This metabolite was not identified in the HLM assay because $\beta$-oxidation mainly occurs in mitochondria, which are not part of the microsomal fraction used in this assay. However, its presence in urine was revealed by the bbCID scan approach for unexpected metabolites, and further characterization (accurate masses and fragmentation pattern) was performed by LC-QToF-MS analysis. A general limitation of pooled HLM assays can be seen in the fact that they do not produce the full human phase I (and II) metabolite spectrum and differ from other in vitro models like hepatocytes [17]. In fact, urine samples (which were available in this study) provide the only valid way for evaluation and confirmation of human SC metabolites suitable as urinary biomarkers-whether predicted by reference spectra of liver microsome assays or by other means [13].

\section{Comparison with metabolic pathways of CUMYL-PEGACLONE}

To detect identical metabolites with the non-fluorinated analog CUMYL-PEGACLONE, a set of six urine samples only positive for metabolites of CUMYL-PEGACLONE was analyzed by the LC-MS/MS method described above. Additionally, the metabolite ranking was compared to the data of the human metabolism study of CUMYL-PEGACLONE [11]. Within this comparative analysis, six identical metabolites (M01, M02, M04, M06, M08, M10) were detected. An

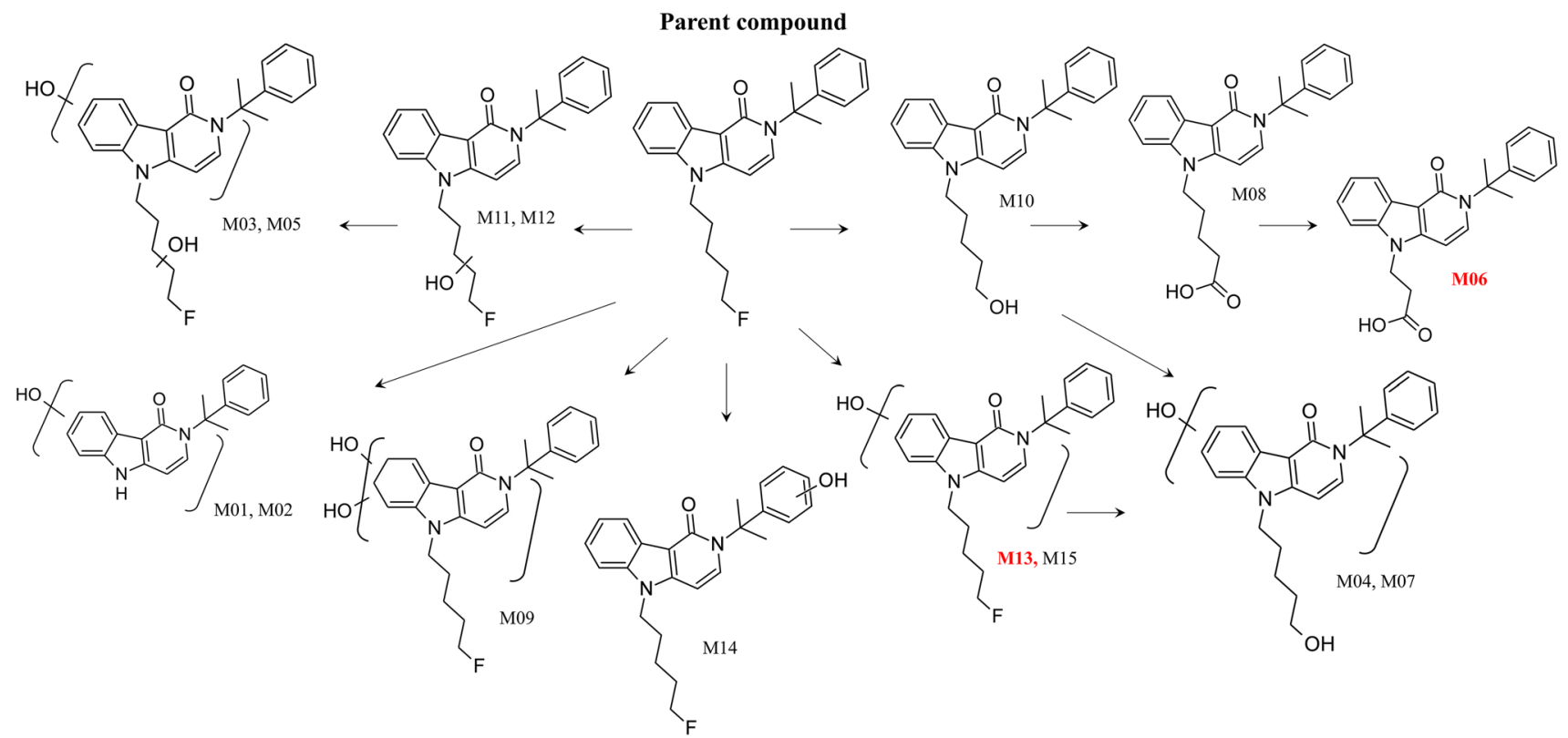

Fig. 3 Postulated human phase I biotransformation pathways of 5F-CUMYL-PEGACLONE for 20 urine samples investigated. Main metabolites suggested as analytical targets for urine analysis are highlighted in red (M06 and M13) 
overlayed chromatogram is shown in the Supplementary Material (Fig. S2).

\section{Evaluation of phase I metabolites as consumption markers}

To evaluate the identified in vivo metabolites as reliable consumption markers, a qualitative ranking of the metabolites by their peak areas was conducted.

In the investigated collected urine samples, M06 was the most abundant metabolite in each of the urine samples. Thus, this main in vivo phase I metabolite can serve as a highly sensitive marker for 5F-CUMYL-PEGACLONE consumption. Because this metabolite was also formed in the metabolism of the non-fluorinated analog (rank position 5 out of 22), selective metabolites must be included in screening methods for unambiguous identification of the consumed compound [11].

M06 is most likely generated by $\beta$-oxidation or oxidative degradation of the 5-fluoropentyl chain. Previous biotransformation steps should include hydrolytic defluorination (M10) and oxidation to the pentanoic acid metabolite (M08). The potential intermediate from oxidative degradation, a butanoic acid metabolite $\left([\mathrm{M}+\mathrm{H}]^{+}\right.$at $\left.m / z 389.1860\right)$, could not be detected in the LC-HRMS bbCID approach. In contrast, for AM-2201, which also carries a 5-fluoropentyl side chain, the butanoic acid metabolite was suggested as a main metabolite [12].

The detection of M13 (overall rank position 3), monohydroxylated at the $\gamma$-carbolinone core with an unaltered 5-fluoropentyl chain, can facilitate a selective detection of 5F-CUMYL-PEGACLONE uptake in urine screening methods. Figure 4 shows the LC-MS/MS EPI spectra of the suggested analytical targets for urine analysis (M06 and M13).
The product of hydrolytic defluorination M10 (rank position 12) was of relatively low abundance in the analyzed set of urine samples. However, M10 was among the major in vitro phase I metabolites (Table S3) and had been tentatively added to the screening method. It was described for several other SCs that the 5-hydroxypentyl metabolite was a common main in vitro and in vivo metabolite of SCs with a 5-fluoropentyl side chain which were prone to hydrolytic defluorination. [12, 23, 24] Surprisingly, this metabolite did not show significant abundances for both of the analogs in human metabolism, which might be explained by quick metabolic oxidation, e.g., to the pentanoic acid metabolite M08 and the propionoic acid metabolite M06.

It should be noted that limitations of a qualitative metabolite ranking based on relative abundances might be biased by matrix effects or many other individual factors like time distance to drug uptake, route of administration and consumption frequency.

\section{Conclusions}

In the present study, the human phase I metabolism of the recently emerged $\gamma$-carbolinone derived SC 5F-CUMYLPEGACLONE was investigated in a collective of human urine specimens. As it has been shown for the non-fluorinated analog CUMYL-PEGACLONE, the drug is subject to extensive metabolism in humans. Metabolic modifications mainly occurred at the $\gamma$-carbolinone core and the 5-fluoropentyl chain. The main in vivo metabolite M06 is an interesting marker for urine analysis when maximum sensitivity is needed (e.g., drug abstinence testing), but this metabolite can also arise from consumption of the non-fluorinated analog CUMYL-PEGACLONE. With the detection of M06

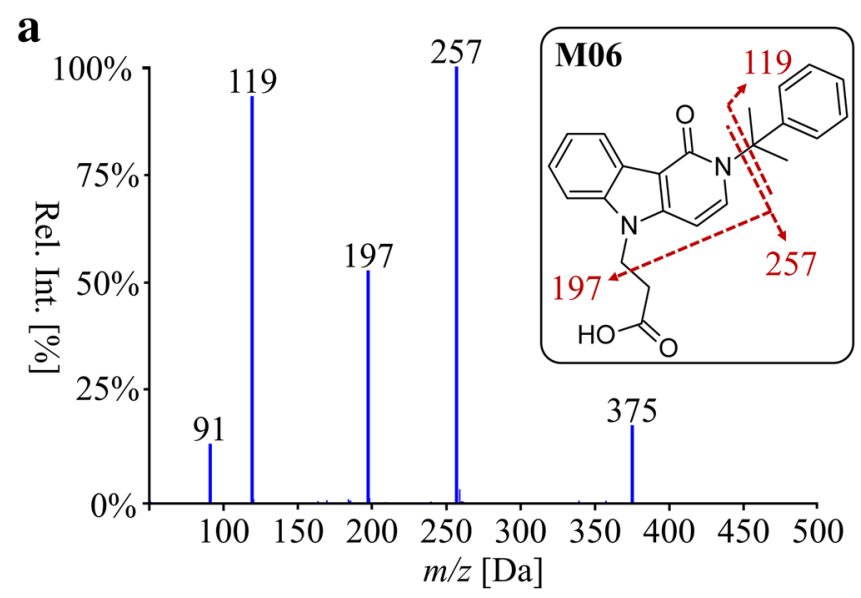

Fig. 4 Enhanced product ion spectra by liquid chromatography-tandem mass spectrometry of the main human phase I metabolites a M06 and b M13. Scans were performed using the optimized declus-

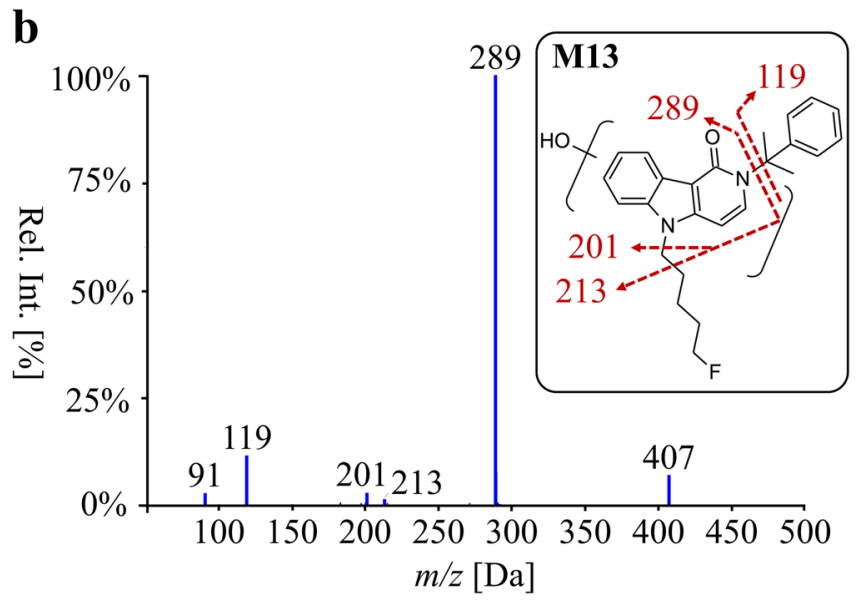

tering potential at $90 \mathrm{~V}$, entrance potential at $10 \mathrm{~V}$ and collision energy at $35 \mathrm{~V}$ with a spread of $\pm 15 \mathrm{~V}$ 
as the main in vivo metabolite, it was shown that untargeted screening approaches are a suitable tool to detect unexpected biotransformation products enhancing the HLM approach. For reliable differentiation between a consumption of both analogs, the metabolite M13 is suggested as a 5F-CUMYLPEGACLONE-specific marker. The suggested marker will allow clinical and forensic toxicologists to specifically prove drug uptake by analysis of urine samples. Although the main metabolite was not generated in the HLM assay, the tentatively implemented microsomal metabolites led to the detection of 20 positive urine samples in routine screening, which were used for further evaluation of the most suitable marker metabolites in urine. This points out that HLM assays offer a practical alternative to other models such as human hepatocytes or fungi in order to generate reference spectra of phase I metabolites. Furthermore, the proposed fragmentation patterns and metabolic pathways might facilitate the detection of other $\gamma$-carbolinone derivatives which might emerge in the near future.

\section{Compliance with ethical standards}

Conflicts of interest The authors declare no competing financial interests.

Ethical approval This article does not contain any studies with human participants or animals performed by any of the authors.

Open Access This article is distributed under the terms of the Creative Commons Attribution 4.0 International License (http://creativeco mmons.org/licenses/by/4.0/), which permits unrestricted use, distribution, and reproduction in any medium, provided you give appropriate credit to the original author(s) and the source, provide a link to the Creative Commons license, and indicate if changes were made.

\section{References}

1. EMCDDA (2018) European drug report 2018: trends and developments. Publications Office of the European Union, Luxembourg. https://doi.org/10.2810/800331

2. Auwärter V, Dresen S, Weinmann W, Müller M, Pütz M, Ferreirós N (2009) 'Spice' and other herbal blends: harmless incense or cannabinoid designer drugs? J Mass Spectrom 44:832-837. https ://doi.org/10.1002/jms.1558

3. Uchiyama N, Kikura-Hanajiri R, Kawahara N, Goda Y (2009) Identification of a cannabimimetic indole as a designer drug in a herbal product. Forensic Toxicol 27:61-66. https://doi. org/10.1007/s11419-009-0069-y

4. Huffman JW, Dai D, Martin BR, Compton DR (1994) Design, synthesis and pharmacology of cannabimimetic indoles. Bioorg Med Chem Lett 4:563-566. https://doi.org/10.1016/S0960 $-894 \times(01) 80155-4$

5. Buchler IP, Hayes MJ, Hegde SG, Hockerman SL, Jones DE, Kortum SW, Rico JG, Tenbrink RE,Wu KK (2009) Indazole derivatives. Patent WO2009/106982

6. Bowden MJ, Williamson JPB (2014) Cannabinoid compounds. Patent WO2014167530 A1
7. Longworth M, Banister SD, Boyd R, Kevin RC, Connor M, McGregor IS, Kassiou M (2017) Pharmacology of cumyl-carboxamide synthetic cannabinoid new psychoactive substances (NPS) CUMYL-BICA, CUMYL-PICA, CUMYL-5F-PICA, CUMYL-5F-PINACA, and their analogues. ACS Chem Neurosci 8:2159-2167. https://doi.org/10.1021/acschemneuro.7b00267

8. Asada A, Doi T, Tagami T, Takeda A, Satsuki Y, Kawaguchi M, Nakamura A, Sawabe Y (2018) Cannabimimetic activities of cumyl carboxamide type synthetic cannabinoids. Forensic Toxicol 36:170-177. https://doi.org/10.1007/s11419-017-0374-9

9. Angerer V, Mogler L, Steitz JP, Bisel P, Hess C, Schoeder CT, Müller CE, Huppertz LM, Westphal F, Schäper J, Auwärter V (2018) Structural characterization and pharmacological evaluation of the new synthetic cannabinoid CUMYL-PEGACLONE. Drug Test Anal 10:597-603. https://doi.org/10.1002/dta.2237

10. Ernst L, Brandhorst K, Papke U, Altrogge A, Zodel S, Langer N, Beuerle T (2017) Identification and quantification of synthetic cannabinoids in 'spice-like' herbal mixtures: update of the German situation in early 2017. Forensic Sci Int 277:51-58. https ://doi.org/10.1016/j.forsciint.2017.05.019

11. Mogler L, Wilde M, Huppertz LM, Weinfurtner G, Franz F, Auwärter V (2018) Phase I metabolism of the recently emerged synthetic cannabinoid CUMYL-PEGACLONE and detection in human urine samples. Drug Test Anal 10:886-891. https://doi. org/10.1002/dta.2352

12. Hutter M, Moosmann B, Kneisel S, Auwärter V (2013) Characteristics of the designer drug and synthetic cannabinoid receptor agonist AM-2201 regarding its chemistry and metabolism. J Mass Spectrom 48:885-894. https://doi.org/10.1002/jms.3229

13. Mogler L, Franz F, Wilde M, Huppertz LM, Halter S, Angerer V, Moosmann B, Auwärter V (2018) Phase I metabolism of the carbazole-derived synthetic cannabinoids EG-018, EG-2201, and MDMB-CHMCZCA and detection in human urine samples. Drug Test Anal. https://doi.org/10.1002/dta.2398

14. Carlier J, Diao X, Scheidweiler KB, Huestis MA (2017) Distinguishing intake of new synthetic cannabinoids ADB-PINACA and 5F-ADB-PINACA with human hepatocyte metabolites and high-resolution mass spectrometry. Clin Chem 63:1008-1021. https://doi.org/10.1373/clinchem.2016.267575

15. Mogler L, Franz F, Rentsch D, Angerer V, Weinfurtner G, Longworth M, Banister SD, Kassiou M, Moosmann B, Auwärter V (2018) Detection of the recently emerged synthetic cannabinoid 5F-MDMB-PICA in 'legal high' products and human urine samples. Drug Test Anal 10:196-205. https://doi.org/10.1002/ dta.2201

16. Watanabe S, Kuzhiumparambil U, Winiarski Z, Fu S (2016) Biotransformation of synthetic cannabinoids JWH-018, JWH073 and AM2201 by Cunninghamella elegans. Forensic Sci Int 261:33-42. https://doi.org/10.1016/j.forsciint.2015.12.023

17. Diao X, Huestis MA (2017) Approaches, challenges, and advances in metabolism of new synthetic cannabinoids and identification of optimal urinary marker metabolites. Clin Pharmacol Ther 101:239-253. https://doi.org/10.1002/cpt.534

18. Franz F, Angerer V, Moosmann B, Auwärter V (2017) Phase I metabolism of the highly potent synthetic cannabinoid MDMBCHMICA and detection in human urine samples. Drug Test Anal 9:744-753. https://doi.org/10.1002/dta.2049

19. Mußhoff F, Skopp G, Pragst F, Sachs H, Thieme D (2009) Guidelines for quality assurance in forensic-toxicological analyses. Toxichem Krimtech 76:142-176 (in German)

20. Kevin RC, Lefever TW, Snyder RW, Patel PR, Fennell TR, Wiley JL, McGregor IS, Thomas BF (2017) In vitro and in vivo pharmacokinetics and metabolism of synthetic cannabinoids CUMYL-PICA and 5F-CUMYL-PICA. Forensic Toxicol 35:333-347. https://doi.org/10.1007/s11419-017-0361-1 
21. Staeheli SN, Poetzsch M, Veloso VP, Bovens M, Bissig C, Steuer AE, Kraemer T (2018) In vitro metabolism of the synthetic cannabinoids CUMYL-PINACA, 5F-CUMYL-PINACA, CUMYL-4CN-BINACA, 5F-CUMYL-P7AICA and CUMYL4CN-B7AICA. Drug Test Anal 10:148-157. https://doi. org/10.1002/dta.2298

22. Shevyrin V, Melkozerov V, Nevero A, Eltsov O, Shafran Y, Morzherin Y, Lebedev AT (2015) Identification and analytical characteristics of synthetic cannabinoids with an indazole3-carboxamide structure bearing a N-1-methoxycarbonylalkyl group. Anal Bioanal Chem 407:6301-6315. https://doi. org/10.1007/s00216-015-8612-7
23. Wohlfarth A, Gandhi AS, Pang S, Zhu M, Scheidweiler KB, Huestis MA (2014) Metabolism of synthetic cannabinoids PB-22 and its 5-fluoro analog, 5F-PB-22, by human hepatocyte incubation and high-resolution mass spectrometry. Anal Bioanal Chem 406:1763-1780. https://doi.org/10.1007/s00216-014-7668-0

24. Diao X, Wohlfarth A, Pang S, Scheidweiler KB, Huestis MA (2016) High-resolution mass spectrometry for characterizing the metabolism of synthetic cannabinoid THJ-018 and its 5-fluoro analog THJ-2201 after incubation in human hepatocytes. Clin Chem 62:157-169. https://doi.org/10.1373/clinchem.2015.243535 